\title{
TIC para la inclusión: una mirada desde Latinoamérica
}

\author{
Julio Cabero Almenara $\left(^{*}\right)$ y Rubicelia Valencia Ortiz $\left(^{* *}\right)$ \\ ${ }^{*}$ ) Universidad de Sevilla - España, (**) Macmillan educación- México
}

\section{RESUMEN}

La Sociedad de la Información y el conocimiento es un fenómeno que impacta a los seres humanos desde hace poco más de cinco décadas. Se caracteriza por la tecnología de la información, que permite su organización en redes que dan a las personas la posibilidad de acceder, compartir y procesar datos, incluso de manera remota y en tiempo real. Las tecnologías digitales permiten superar los límites del intelecto humano y se da una interacción constante entre la tecnología y la sociedad, caracterizadas por la globalización y la digitalización.

La difusión del uso de las tecnologías de la información y la comunicación (TIC) ha sido paulatina dentro de las sociedades, y entre los países más desarrollados y los de menor desarrollo, existe la tendencia constante hacia la inclusión, lo que repercute en los ámbitos laboral, político, económico y social y educativo, siendo este último el enfoque de este capítulo.

En este artículo se propone la incorporación de las TIC a las sociedades latinoamericanas es una labor política y tecnológica, que no se limita alavinculación entre tecnología y educación, pues esta última debe reforzarse mediante la adecuación de las prácticas educativas y el cultivo de competencias entre docentes y alumnos.

Palabras Clave: Tecnología, información, comunicación, inclusión, educación.

\section{ITC for inclusion: a look from Latin America}

\section{ABSTRACT}

Information and knowledge society has had an impact on human beings since fifty years ago. It is caracterized by information technology that allows data be organized in nets that give to the people the opportunity to access, deliver and share data, in a remote way and real time. Digital technologies allow us to exceed the limits of human intelect and the constant interaction among technology and society, and are characterized by globalization and digitalization.

Even though the spreading of information and communication technology has been gradual among societies, and in the same wayamong developed and underdeveloped countries, there is a continuous trend towards inclusion, that has an effect on the fields of labor, politics, economics, society and education.

This article lays out incorporation of Information and Communication Technologies into Latin American societies is a political as well as a technological task, not restricted to the links between technology and education, that may be reinforced by adequating educational practices and competences among teachers and students.

Keywords: Technology, information, communication, inclusion, education.

\section{El escenario de la Sociedad de la Información/Conocimiento.}

Los orígenes de la Sociedad de la Información y el Conocimiento (SIC) se sitúan en los años 60, ofreciéndose sobre ella diversas definiciones que giran alrededor de indicar que es un nuevo estadio de desarrollo social, que supera a los anteriores, agrícola e industrial y postindustrial, y que viene determinado por la capacidad que tienen las personas e instituciones por obtener, compartir y procesar cualquier información, desde cualquier lugar y en una diversidad de formatos y sistemas simbólicos.

Sociedad que se caracteriza por una serie de hechos distintivos: globalización económica y cultural, velocidad de genera- ción y transforma de la información, el aprendizaje constante, la ruptura de los conceptos tradicionales de espacio y tiempo, ser una sociedad que requiere el aprendizaje constante, ser una sociedad de redes, apoyarnos en modelos económicos centrados en la información, y la amplitud de Tecnologías de la Información y Comunicación (TIC) que tenemos a nuestra disposición. Siendo posiblemente ésta última, una de sus características distintivas.

Tal situación nos introduce en un contexto ambiguo, complejo, dinámico y desconocido, diferente al de la sociedad postindustrial, donde todo tendía a estar regulado y estandarizado. Al respecto Bauman (2007) indica que estamos en un mundo de modernidad líquida donde todo es rápido y perenne, o a Lipo- 
vetsky (2016) señalar que nos desenvolvemos en una sociedad de la ligereza.

En una línea similar Valencia (2018) indica que "vivimos en un mundo VUCA (Volatility, Uncertainty, Complexity, Ambiguity, por sus siglas en inglés, o sea, Volátil, Incierto, Complejo y Ambiguo), con dinámicas de cambio constante y difícil de predecir, con multiplicidad de fuerzas que se interrelacionan en todos los ámbitos del conocimiento y la vida, con una interdependencia de variables difíciles de descifrar, y con oportunidades y desafíos permanentes."

Estamos por tanto en una situación de cambio constante respecto a los estadios anteriores. Cambios que se producen en diferentes sectores: sociales, políticos, económicos y laborales. Para West (2016) es una época de "megacambio", lo que repercute en que los patrones sociales, económicos y políticos han dejado de ser fijos, y ello produce inseguridad y miedoa los otros y al futuro. Tal situación nos acercará cada vez más a ser nómadas del conocimiento y tener que desenvolvernos en un contexto del aprendizaje constante.

Y en este entramado de velocidad y cambio las tecnologías jugarán un papel determinante ya "acelera o multiplica los efectos de la globalización. La mejora de las telecomunicaciones, la conectividad, internet, etc., alimentan la globalización económica, política y cultural. Por otra parte, los mercados globalizados son el marco natural de la revolución tecnológica, en el que puede desarrollar todas sus potencialidades." (González, 2017, p. 15).

Brynjolfsson y McAfee (2014) llaman a esta época la "segunda edad de las máquinas", que presenta una diferencia fundamental con respecto a la primera, ya que no se basa en las máquinas de vapor para superar los límites físicos de los humanos y los animales, sino que se apoya en las tecnologías digitales para superar los límites de las capacidades intelectuales humanas.

Como señalan Cummings Regeer, De Haan, Zweekhorst y Bunders (2018), en el análisis que realizan de los discursos que se efectúan sobre la justificación de las tecnologías en la SIC; suelen darse dos propuestas para su justificación, que tienen cargas ideológicas diferentes: a) un discurso tecno-científico-económi$\mathrm{co}$, en gran parte propuesto por gobiernos de países desarrollados, que plantea que el conocimiento científico y tecnológico condiciona directamente el desarrollo económico y social de las sociedades modernas, lo que le otorga un marcado carácter determinista y estandarizador, y b) otro más pluralista-participativo que tiende a otorgarles un rol más inclusivo de las mismas en la sociedad, favoreciendo los saberes locales y al desarrollo endógeno y significativo de la persona y máximo respeto por la diversidad lingüística y cultural.

En una, es la tecnología, y sus supuestas potencialidades instrumentales las que determinarán su utilización e implicación, y en la otra, son las personas con sus características cognitivas, actitudinales y grado de aceptación de las tecnologías, las que determinarán su grado de incorporación social, personal y cultural. Es precisamente la segunda, la que permitirá resolver el "solucionismo tecnológico" al que se ha referido Morozov (2013); lo que nos llevará a asumir desde el principio que los cambios que se necesitan en la SIC, no sean solo tecnológicos, sino también organizativos, sociales, culturales y políticos.

En este escenario, la tecnología y la sociedad están interactuando constantemente, de manera que se han convertido en una de las variables críticas de los momentos sociales actuales, y que de forma segura nos desenvolveremos en los escenarios de la cuarta revolución industrial,donde las tecnologías tomarán mayor protagonismo incluso a nivel inteligente (Floridi, 2014).

Dos características básicas de la sociedad actual son la globalización y la digitalización (Lledó, 2018). Respecto a la primera surgen voces críticas respecto a ese mundo de crecimiento global y de consolidación de los sistemas democráticos que se nos vendía a través de ella, y empiezan a existir temores por la pérdida y desaparición de puestos de trabajo o el aumento de la precariedad salarial (González, 2017). Y la segunda, nos está llevando a sociedades donde al menos el 70\% del PIB "depende de los bienes intangibles, que están relacionados con la información, más que con los bienes materiales, que son el producto físico de los procesos agrícolas o de fabricación. Sus economías dependen en gran medida de activos basados en información (economía basada en el conocimiento), servicios intensivos en información (especialmente servicios comerciales y de propiedad, comunicaciones, finanzas, seguros y entretenimiento), y sectores públicos orientados a la información (especialmente educación, administración pública y cuidado de la salud)." (Floridi, 2014, p. 4)

Pero no debemos olvidarnos de que si las tecnologías nos abren grandes posibilidades para la comunicación, interacción y colaboración de las personas, al mismo tiempo se convierten en un fuerte desafío para el trabajo, la libertad y la igualdad (Harari, 2018).

También debemos ser conscientes que los cambios y transformaciones tecnológicasafectan "a nuestro sentido del yo, cómo nos relacionamos con los demás y cómo damos forma e interactuamos con nuestro mundo" (Floridi, 2017, p. 8). Y apuntan por tanto cambios más significativos que los económicos e implican fuertes beneficios para el empoderamiento de la persona.

La significación de las TIC, hace que su no acceso se convierta en motivo de exclusión social, de ahí que en los últimos años se estén adoptando medidas por parte de los gobiernos nacionales y autonómicos, para fomentar su presencia y la capacitación digital de sus ciudadanos.

\section{Hacia una sociedad democrática: la inclusión educativa.}

Hablar de inclusión es referirnos a un término que presenta diferentes significados:

- a la discapacidad y a las necesidades educativas especiales;

- respuesta a las exclusiones disciplinarias;

- referida a los grupos vulnerables de exclusión;

- y potenciación de una escuela para todos (Ainscow y Miles, 2009).

La última perspectiva, es tener en cuenta diferentes aspectos:

- Es no creer que sus únicos receptores son las personas con alguna discapacidad;

- Es referirnos al derecho que tienen todas las personas a la educación, y la incorporación de aquellas personas que son excluidas del sistema educativo por aspectos tales como su género, su cultura, su raza o sus características personales;

- Es centrarnos no solo en las personas discapacitadas, sino también hacerlo de las que viven en la pobreza y la marginalidad, de las que pertenecen a etnias minoritarias, de las que hablan lenguas minoritarias o de las que viven en las zonas periféricas con falta de recursos.

Hablar de escuela inclusiva es referirnos a una escuela "que no pone requisitos de entrada ni mecanismos de selección o discriminación de ningún tipo para hacer, realmente efectivos los derechos a la educación, a la igualdad de oportunidades y a la participación" (Peñafiel, 2014, p. 29). Es movernos en la idea de la educación como derecho de las personas (Moya, 2015), y por tanto de su percepción como instrumento facilitador de la igualdad de oportunidades. 
Los términos inclusión e integración se refieren a aspectos diferentes; con el primero nos centramos en el alumno, y con el segundo, en el aula, preocupada por las variables del enseñanza-aprendizaje, centrada en las dificultades que surgen en la colaboración, instaurar estrategias para los docentes, proponer un entorno de clase que favorece la adaptación y brinde apoyo con regularidad, y propiciar estructuras organizativas que faciliten con flexibilidad la incorporación de la persona (Escribano y Martínez, 2013). Por tanto, referirnos a la inclusión y no a la integración, es pasar de un modelo individual, donde la dificultad o limitación está en el individuo, y es él como señala Casanova (2017), el que debe hacer el esfuerzo para integrarse al contexto; a uno social, donde las dificultades y las limitaciones están en la sociedad, y por tanto el esfuerzo a realizarse pasa del sujeto a la institución, por tanto ella deberá adoptar las medidas suficientes para facilitar la incorporación de la persona, independientemente de sus características, etnias, religión, género,...

El término educación inclusiva es por tanto más amplio que el de integración y parten de supuestos diferentes; la inclusión persigue mejorar la calidad educativa de todos los miembros de la comunidad escolar: alumnos, familia, comunidad,... (Peñafiel, 2014). Es hablar, por una parte, de una educación para todos: "la educación inclusiva tiene que ver con cómo, dónde, por qué y con qué consecuencias educamos a todos los alumnos" (Escribano y Martínez, 2013, p. 24), y por otra de la educación como derecho humano, de la búsqueda de una educación de calidad y de una educación con fuerte sentido social (Moliner, 2013).

La educación inclusiva es un proceso que responde a la diversidad de necesidades de todos los alumnos, a través de las prácticas en las escuelas, las culturas y las comunidades, reduciendo así la exclusión dentro de la educación. Implica al mismo tiempo, cambios y modificaciones en el contenido, los enfoques, las estructuras, las estrategias de enseñanza y las modalidades organizativas, todo ello a través de una visión común que alcance a todas las personas de la misma edad, y con la convicción de que es responsabilidad del sistema educativo ordinario educar a todos ellos, dándoles las mismas oportunidades con garantías de calidad y gratuidad. Como señala Barrio (2009, p. 14): "su supuesto básico es que hay que modificar el sistema para responder a todos los alumnos, en vez de entender que son los alumnos quienes se tienen que adaptar al sistema, integrándose en él."

Como ya señalaron Cabero y Córdoba (2009), tanto el objetivo como el centro de preocupación de la inclusión e integración son diferentes. En lo que se refiere a su objetivo, el de la inclusión es de mayor magnitud que el de la integración, en el sentido de que la última se centra en el colectivo de los discapacitados, y la primera en todos los estudiantes. Por otra parte, mientras el centro de preocupación de la integración es mejorar y cambiar la educación especial, el de la inclusión es perseguir la transformación de la cultura, la organización educativa y la práctica escolar. Algunos autores incluso llegan a señalar que "la inclusión no es un mero enfoque en educación, sino una reorientación respecto a los errores de la "integración escolar" (Castro y Blanco, 2009, 94). Perspectiva que ha ido ocupando progresivamente parcela de interés en la investigación (Suárez y López, 2018).

Por tanto, debemos percibirla como una estrategia para minimizar o eliminar las barreras que dificultan la incorporación de todas las personas a diferentes sectores sociales, que van desde los económicos, formativos, sociales, culturales o políticos.

Finalmente señalar las líneas que Marchesi, Blanco y Hernández (2014) establecen como "hoja de ruta" para alcanzar sistemas educativos inclusivos: 1) aumentar la cobertura y mejorar la calidad de la educación de la primera infancia que se brinda a los grupos en situación de mayor vulnerabilidad; 2) asegurar la progresión oportuna y la conclusión universal de la educación primera y secundaria; 3) eliminar las diferentes formas de discriminación para hacer efectivo el derecho a la educación; mejorar la calidad de los procesos educativos y entonos de aprendizaje para cerrar las brechas en el acceso y la aparición del conocimiento; 5) invertir más en los docentes y desarrollar políticas que integren la formación inicial y en servicio, la inserción laboral y condiciones adecuadas de trabajo; 6) desarrollar sistemas de apoyo que colaboren con las escuelas y docentes en la atención a la diversidad del alumnado; 7) fortalecer los sistemas integrales de protección y promoción social; 8) democratizar el acceso a las TIC; 9) aumentar la inversión y hacer más equitativo el gasto público en educación; y 10 ) desarrollar sistemas de información desagregada por factores de exclusión.

\section{TIC y educación inclusiva.}

Como se ha señalado lainclusión parte de la idea clave, de quecon la intervención de todas las personas en el procesode enseñanza-aprendizaje y mediante la utilización de técnicas de enseñanza concretas, es posible atender atodas ellas y llevarlas a situaciones de excelencia educativa. Y es desde estaopción, de las cual las TIC pueden ser de granayuda, para tratar de favorecer el acercamientode todas las personas a una formación decalidad y no discriminante.

Las relaciones entre las TIC y la educación inclusiva pueden entenderse desde dos perspectivas: a) que con suutilización se puede favorecer el alcanzaruna educación de calidad, y eliminar lasbarreras que impiden el acercamiento detodas las personas a la cultura y la educación, y b) que con su diseño y estructura se pueden crear tanto entornosaccesibles para la persona como entornos que dificulten el acceso a los mismos; es decir, con ello podemos o facilitar la inclusión o potenciar la exclusión. Y de esta última forma que sirvan como instrumentos potenciadores del aumento de la brecha digital entre colectivos (Cabero y Ruiz Palmero, 2018).

Para propiciar que las TIC favorezcan la inclusión debemos contemplar una serie de elementos:que sean éticos, que se centralicen en la persona, que potencien la participación, que se adapten a las características de diferentes personas y colectivos, que sean interoperables y que faciliten su interconexión con otros, asequibles en su coste, que sean sostenibles y en consecuencia tengan un fácil mantenimiento, replicables, seguros y accesibles (Valero, 2010). Lo que estamos comentando se refiere directamente a la temática de pensar en la accesibilidad en su diseño es de capital importancia, ya que pensar en accesibilidad que permite la adaptación de las tecnologías a las preferencias de cada estudiante. Y en este sentido debemos reconocer que las instituciones educativas están haciendo fuertes esfuerzos en sus sitios oficiales (Toledo, Sánchez y Gutiérrez, 2013). Para Alba (2016, p. 16): “La clave está en el diseño, en lograr un diseño curricular en el que los objetivos, la forma de evaluación, los métodos y los materiales permitan que cada estudiante pueda acceder, participar y aprender en los contextos de enseñanza a los que pertenecen."

Un movimiento de gran importancia en el terreno del diseño, es el denominado “Diseño Universal para el Aprendizaje” (DUA) (Universal Design for Learning), que como apuntan Escribano y Martínez (2013, p. 87) se refiere a un "paradigma relativamente nuevo dirigido al desarrollo de productos y entornos de fácil acceso para el mayor número de personas sin la necesidad de adaptarlos o rediseñarlos de una forma especial"; el cual se apoya en una serie de principios: igualdad de uso, flexibilidad, ser simple e intuitivo, que se perciba la información de manera fácil, que sea tolerante a los errores que puedan comentar las personas 
en su utilización, que requieran escaso esfuerzo físico, y que posea las dimensiones apropiadas (Escribano y Martínez, 2013, pp. 87-88). Por su parte el "National Center on Universal Design for Learning" llama la atención respecto a que el DUA, se apoyarnos en tres principios para proporcionar múltiples medios para: 1) la representación (el qué del aprendizaje; 2) para la acción y la expresión (el cómo del aprendizaje); y 3) los compromisos (el "por qué" del aprendizaje).

No debe caber la menor duda, que losproyectos TIC orientados a colectivos vulnerables, representan una excelente alternativa educativapara facilitar su inclusión en la SIC, ya que favorecen la creaciónde escenografías enriquecidas de aprendizaje, queayudan a superar los déficits sociales, económicos, escolaresy culturalesde la persona, todo ello propiciando la equidad en el acceso ala calidad del aprendizaje y potenciando quelas personas puedan acceder a conocimientos noubicados en su contexto cercano.

Reclamar unas TIC accesibles e inclusivas, es sinónimo de querer garantizar una senda flexible a ellas para todas las personas, independientemente de sus características y posibilidades de acceso; de manera que todos puedan beneficiarse de las posibilidades que ofrecen para presentar la información en diferentes formatos, interaccionar con personas independientemente del espacio y el tiempo en el cual se encuentren, convertirse en productores de mensajes, o acceder a entornos formativos de calidad.

Relacionado con el tema que analizamos se encuentra la problemática de la brecha digital, que es la "diferenciación producida entre aquellas personas, instituciones, sociedades o países, que pueden acceder a las TIC de forma general y a Internet de manera particular, y aquellas que no pueden hacerlo; es decir, puede ser definida en términos de la desigualdad de posibilidades que existen para acceder a la información, al conocimiento y la educación mediante las TIC, sea por motivos económicos, de edad, de género, raza, ubicación geográfica, u otros motivos." (Cabero y Ruiz-Palmero, 2018, p. 24).

Hablar de brecha digital, es tener presente que nos encontramos con diferentes tipos (Van Deursen y Van Dijk, 2014; Cabero y Ruiz-Palmero, 2018); normalmente se entendía que la brecha digital era consecuencia de una brecha económica y social, y lo es y sigue siendo, pero la brecha se ha ampliado, a una brecha de segundo y tercer orden, dondeteniendo las personas acceso físico a las tecnologías, no las utilizan por no saber qué hacer con ellas, o los usos que hacen con ellas son muy elementales y de poca riqueza conceptual, como consecuencia del nivel de formación y competencia digital que posean. La primera se aborda con medidas económicas, las otras, con medidas de políticas educativas; las primeras son más fáciles de resolver, las segundas más complejas, y requieren movernos en una concepción de inclusión educativa, y de que la educación es un derecho democrático de la persona.

Para finalizar este apartado, y relacionado con la utilización de las tecnologías en relación con la inclusión educativa, es necesario comenzar a percibir tres perspectivas diferentes de concreción: TIC (Tecnologías de la Información y Comunicación), TAC (Tecnologías para el Aprendizaje y el Conocimiento) y TEP (Tecnologías para el Empoderamiento y la Participación) (Cabero, 2014).

Desde la primera las entendemos como recursos facilitadores y transmisores de información y recursos educativos para los estudiantes. Desde el enfoque como TAC, se sugiere su utilización como instrumentos facilitadores del aprendizaje y la difusión del conocimiento, son percibidas por tanto como herramientas para la realización de actividades para el aprendizaje y el análisis de la realidad circundante por el estudiante. Y desde la última de las perspectivas, se trataría de concebirlas como instrumentos para la participación y la colaboración de las personas.

Finalmente señalar que ha existido una línea de trabajo para la incorporación de las TIC para las personas con discapacidad, en función de las potencialidades que estas ofrecen, y que cuando se han desarrollado metaanálisis de las experiencias realizadas (Orozco, Tejedor y Calvo, 2017) han demostrado su eficacia. Si bien debemos reconocer que se ha impulsado más desde la práctica que desde la propia investigación, no existiendo una fuerte presencia respecto a otras áreas de investigación educativa (Starcic y Bagon, 2014; Suárez y López, 2018).

Las posibilidades que las TIC ofrecen a las personas con discapacidad son amplias: facilitar el acceso a la información y su acceso en diferentes soportes y códigos, adaptación del entorno a las necesidades de la persona, facilitar el acceso al mundo laboral, aumentar la autonomía y la independencia de la persona, favorecer un modelo de comunicación y de formación multisensorial, favorecer un mayor control de las situaciones sociales, superar el estigma de la discapacidad, aumentar los sentimientos de seguridad, ampliar los canales de comunicación, y suplantar determinadas habilidades cognitivas que la persona por sus características no sea capaz de realizar (Cullen y Alber-Morgan, 2015; Fernández-Batanero, Reyes y El Homran, 2018; CEPAL, 2018. Aunque debemos reconocer que todavía la incorporación de recursos para estas personas en los centros es más bien limitada (Arnaiz, de Haro y Maldonado, 2019).

\section{TIC e inclusión educativa: miradas desde Latinoamérica.}

Para la Unesco (2017): “la evidencia muestra que la participación en esta cultura no se distribuye en términos equitativos tanto en lo que refiere a las oportunidades de acceso como a las capacidades de uso y aprovechamiento de las nuevas tecnologías. Esta situación constituye un riesgo no sólo de mantener las brechas sociales existentes, sino que podría estar amplificándolas." (Unesco, 2017, p. 3). De ahí que la misma institución reclame sea necesario adoptar dos medias básicas en Latinoamérica: aumentar el número de ordenadores conectados a Internet y mejorar la conectividad a Internet. Medidas que deben fundamentalmente llevarse a cabo "en los sectores más vulnerables, y con un énfasis especial en la población de niveles socioeconómicos más bajos y los que habitan en sectores rurales." (Unesco, 2017, p. 3).

Esta significación ha repercutido para que en los últimos años se hayan realizado fuertes intentos y declaraciones para favorecer y propiciar la presencia de las TIC. Así entre los objetivos de la agenda digital para América Latina y el Caribe (eLAC2018) se señalaban tres relacionados con la temática de nuestro trabajo:

14: Incorporar o fortalecer el uso de las TIC en la educación y promover el desarrollo de programas que contemplen la capacitación de docentes, nuevos modelos pedagógicos, la generación, adaptación e intercambio de recursos educativos abiertos, la gestión de las instituciones de educación y evaluaciones educativas;

17: Promover una perspectiva integral de igualdad de género en las políticas públicas de desarrollo digital, asegurando el pleno acceso y uso de las TIC para las mujeres y niñas, e impulsando el rol de participación y liderazgo de las mujeres en espacios públicos y privados de decisión en materia digital.

18: Asegurar el acceso a las TIC a los grupos vulnerables, para mejorar su inserción social, educativa, cultural y económica. 
O como entre las Metas 2030 para Latinoamérica (CEPAL, 2018), dos se refieren a nuestra temática:

Meta 5: Lograr la igualdad de género y empoderar a todas las mujeres y las niñas" 5.b ("Mejorar el uso de la tecnología, en particular la tecnología de la información y las comunicaciones, para promover el empoderamiento de la mujer");

Meta 9: Construir infraestructurasresilientes, promover la industrialización inclusiva y sostenible y fomentarla innovación). Aumentar de forma significativa el acceso a la tecnología de la información y las comunicaciones y esforzarse por facilitar el acceso universal y asequible a Internet en los países menos adelantados a más tardar en 2020 .

No obstante, los países de la región continúan avanzando a velocidades distintas, con brechas dentro de ellos y entre ellos, y con diferenciasrespecto a las economías más desarrolladas" (CEPAL, 2015, p. 2). Y la realidad es que las diferencias entre países desarrollados y los que están en vías de desarrollo han variado relativamente poco. En concreto, actualmente, mientras en los países desarrollados cerca del $80 \%$ de los hogares cuenta con acceso a un ordenador o Internet, en los países en vías de desarrollo esta cifra se reduce a la mitad. El porcentaje de alumnos que declara que tiene un ordenador para estudiar está en Latinoamérica, está por debajo de la media de la OCDE (91), los de mayor presencia en Latinoamérica son Uruguay (89) y Perú (55). Al mismo tiempo se dan fuertes diferencias entre las escue- las urbanas y rurales (Unesco, 2017). Y asumiendo los diferentes esfuerzos realizados siguen existiendo, como señala el CEPAL (2016) dificultades relacionadas con la calidad y equidad en el acceso a Internet en y entre los países Latinoamericanos.

Pero si ha existido un esfuerzo en aumentar la presencia de las TIC, también se ha dado otro en potenciar la atención a las personas con discapacidad y en situaciones de exclusión. Ello ha llevado a que se hayan efectuado esfuerzos hacia la protección y promoción de los derechos e inclusión de las personas con discapacidad. Como señala CEPAL (2018, p. 9): “los 19 países latinoamericanos y 11 países del Caribe han ratificado la Convención sobre los derechos de las personas con discapacidad (CRPD). Los países de la región han tratado de implementar la CDPD mediante la adopción de legislación a nivel nacional, el establecimiento de institutos y consejos nacionales y la implementación de estrategias, políticas y programas." Una revisión deestosmarcos legislativo puede observarse en el reciente trabajo de Ullman (2017).

Pero aun reconociendo los avances efectuados, el uso de Internet es diferente entre las personas con y sin discapacidades, siendo menor en las primeras; dándose también esa brecha entre la población con discapacidad. Brechas que son especialmente graves para las personas con niveles más bajos de educación, las sin trabajo, los mayores con discapacidades y las mujeres con discapacidad (CEPAL, 2018).

Como señalamos se han desarrollado diferentes planes de incorporación de las TIC, especialmente internet y ordenadores, en distintos países Latinoamericanos, en la tabla $n^{-}$1, se presentan algunos de estos proyectos.

Tabla 1.

Programas de incorporación de las TIC en Latinoamérica.

\begin{tabular}{|c|c|c|}
\hline Nombre & País & Características \\
\hline Plan Ceibal & Uruguay & https://www.ceibal.edu.uy/es \\
\hline Conectar igualdad & Argentina & https://www.argentina.gob.ar/educacion/aprender-conectados/conectar-igualdad \\
\hline $\begin{array}{l}\text { Una niña. Un niño. } \\
\text { Una computadora }\end{array}$ & El Salvador & http://www.cienciaytecnologia.edu.sv/programas/ppc.html \\
\hline$@$ @prende.mx & México & http://www.aprende.edu.mx \\
\hline Canaima educativo & Venezuela & https://wiki.canaimaeducativo.gob.ve/doku.php \\
\hline Me conecto para aprender & Chile & http://meconecto.mineduc.cl/ \\
\hline Um computador por aluno & Brasil & $\begin{array}{l}\text { https://www.fnde.gov.br/programas/proinfo/eixos-de-atuacao/projeto-um-computa- } \\
\text { dro-por-aluno-uca }\end{array}$ \\
\hline $\begin{array}{l}\text { Computadores para Educar } \\
\text { Colombia aprende }\end{array}$ & Colombia & $\begin{array}{l}\text { http://www.computadoresparaeducar.gov.co/ } \\
\text { http://aprende.colombiaaprende.edu.co/cainicio }\end{array}$ \\
\hline Una lapto por Niño & Perú & http://repositorio.minedu.gob.pe/handle/123456789/800 \\
\hline $\begin{array}{l}\text { Proyectos de la Fundación } \\
\text { Omar Dengo }\end{array}$ & Costa Rica & http://www.fod.ac.cr/index.php?option=com_content\&view=article\&id=5\&Itemid=103 \\
\hline República Digital & República Dominicana & https://republicadigital.gob.do/ \\
\hline
\end{tabular}

Los alcances de los proyectos han sido diferentes, pero en líneas generales han seguido las propuestas de los "Planes Maestros para la adaptación de la Educación a las TIC" de Korea, el Programa OLPC (One Laptop per Child), y las aportaciones que se iban extrayendo del "Plan Ceibal" de Uruguay, que ha sido uno de los más constantes y exitoso.

Aunque los programas de un "Ordenador por niño" no siempre han resultado exitosos (Zheng, Warschauer, Lin y Chang, 2016), entre otros motivos por su carácter economicista, y poco flexible y estandarizante. Tampoco podemos negar que las experiencias a pequeña escala si han dado resultados positivos, sobre todo cuando los esfuerzos se realizaban no solo en dotar de infraestructuras tecnológicas, sino que además se intervenía con los profesores, alumnos y ciudadanos para la apropiación de la tecnología.

Por lo general los proyectos se articulan alrededor de cuatro objetivos: facilitar a las instituciones educativas de dispositivos tecnológicos, mejorar las infraestructuras de telecomunicación, formar a los profesores, y producir contenidos digitales; como ocurre con el Plan Ceibal ("Conectividad Educativa de Informática Básica para el Aprendizaje en Línea") (Rivera y Cobos, 2018). Aunque algunos como el "Plan Aprender conectado", el 
“México @prende.mx" o "República Digital”,tienden también a incorporar en sus prioridades la formación de los alumnos los lenguajes informáticos, la programación, la robótica y el pensamiento computacional (Ministerio de Educación de la Nación, 2017; Cárdenas y Anaya, 2018).

Algunos de los errores que se han cometido, y se están cometiendo en la incorporación de las TIC, es la visión que se tiene de los docentes como meros replicadores y utilizadores de ellas. Como se señala en la estrategia “@prende.mx": “Bajo este Modelo se integran la tecnología mediante promotores que impulsen su uso en las aulas de medios; ofreciendo al personal docente soluciones tecnológicas útiles para gestionar su clase y eficientar procesos administrativos, evaluar al alumnado y monitorear la efectividad de la autonomía en las escuelas." (Cárdenas y Anaya, 2018, p. 37).

Visión exitosa, es la propuesta en el Plan “@prende.mx" cuando se señala el "- adoptar una estrategia de implementación en fases que considere la introducción de dispositivos con un criterio geográfico único y de acuerdo con el grado de preparación o demanda de cada escuela. El propósito es que las escuelas que generen buenas prácticas beneficien a las escuelas menos preparadas, una vez que adopten la iniciativa en etapas subsecuentes." Si bien tales aplicaciones, no deben ser miméticas sino contextualizadas.

Es importante destacar errores que se cometieron en las primeras implantaciones, como el centrarse exclusivamente en la dotación de recursos y mejora de las infraestructuras, se fueron progresivamente resolviendo en las sucesivas implantaciones y se establecieron medidas tanto para la formación del profesorado como para el monitoreo y seguimiento de su implantación. Lo que ha permitido disponer de datos para reorientar los proyectos y acoplarlos a los objetivos que se habían marcado (Fundación Telefónica, 2018).

Por lo que se refiere a la formación del profesorado, tenemos que señalar dos aspectos, por una parte que si la formación del profesorado es deficitaria en lo que se refiere a la incorporación de las TIC de manera general en el caso de su incorporación en situaciones de inclusión educativa es más deficitaria aún (Fernández-Batanero, Román y El Homrani, 2017; Fernández-Batanero, Reyes y El Homrani, 2018; Martínez, Gutiérrez y Fernández, 2018; Tello y Cascales, 2015), situación que se ve reforzada con la baja formación que para ello reciben los maestros en formación (López, Vázquez y Sarasola, 2015; Pegalajar, 2017). Es más, algunas veces incluso cuando se alude a las competencias TIC que deben tener los docentes, no se alude en ningún momento a competencias para su integración con personas con diferentes tipos de discapacidad y para la inclusión educativa (Rangel, 2015), o que los profesores perciben la no necesidad de estar formado para ello (Ortíz, Almanzan, Peñaherrera y Cachón, 2014).

Ahora bien, desde nuestro punto de vista no es suficiente con reclamar y poner en funcionamiento planes de formación del profesorado en TIC, sino el solicitar que se lleven a cabo desde presupuestos diferentes a los tradicionalmente desarrollado; es decir, centrándose menos en las dimensiones instrumentales y abordando más las dimensiones pedagógicas y didácticas (Cabero y Valencia, 2018), de empoderamiento de la persona y de adaptabilidad a sus características y necesidades individuales.

\section{A manera de conclusión.}

Se observa, por una parte, un fuerte avance de los planes de incorporación de las TIC, lo que significa la importancia de éstas TIC están teniendo para los países de Latinoamérica;pero al mismo tiempo se encuentra la falta de políticas tanto para la evaluación de su implantación, como para su seguimiento; de ahí que en muchos países vayan apareciendo y desapareciendo sucesivos programas. Ello hace necesario, establecer niveles progresivos de incorporación de los programas, a corto, medios y largo plazo; y se centrarse muchas veces no en la presencia de equipos y si en mejorar la calidad y potencialidad de la conectividad.

Las transformaciones, para cambiar la educación, no son meramente tecnológicas sino radicalmente educativas. El informe PISA (OCDE, 2015), puso claramente de manifiesto que, si los proyectos de incorporación de TIC han reducido, o por lo menos mantenido, la denominada primera brecha digital, la segunda referida a los usos se sigue manteniendo, de ahí la necesidad de que, para favorecer la inclusión mediante las TIC, primero es necesario que los alumnos tengan un mínimo de competencias instrumentales como la lectura y las matemáticas.

Los datos apuntados indican que no es suficiente con la dotación de las TIC a los centros, sino que se debe adoptar al mismo tiempo fuertes medidas en la formación del profesorado, y que la misma se lleve desde perspectivas diferentes a las instrumentales para que se facilite su adopción por los docentes para propiciar la transformación del sistema educativo. Por tanto, se trata de que los planes para su incorporación de TIC, no se centren en ellas, sino se concentren en la transformación de las prácticas educativas a través de ellas; y para ello pudiera ser interesante insistir en la formación inicial de los docentes. Son los usos de las tecnologías los que determinan la transformación de las prácticas educativas, y es en este sentido donde se deben empezar a adoptar medidas.

Formación que debe tener una especial atención en el uso de las TIC para personas con diferentes tipos de discapacidades y para colectivos en riesgos de exclusión.

Se hace también necesario la potenciación de la producción de objetos de aprendizaje digitales, que puedan ser utilizados en abierto, para que sea el docente y el estudiante, el que lo contextualice en función de sus necesidades educativas.

Deben adoptarse medidas para que la utilización de las TIC no se centre exclusivamente en el aula, sino que alcancen a utilizaciones fueras de ellas, ya que estos usos serán los que empoderen a los colectivos en vías de exclusión a facilitarles su incorporación a la partición social, y se beneficien de las posibilidades que tienen en sectores como los laborales, de salud, y de relación de la ciudadanía con la administración.

Finalmente señalar que todo programa que se ponga en acción debe tender a alcanzar las dimensiones que la Unesco estableció para alcanzar la calidad de los programas formativos: relevancia, equidad, pertinencia, eficacia y eficiencia (Severin, 2016).

\section{Referencias}

Ainscow, M. y Miles, S. (2009). Desarrollando sistemas de educación inclusiva. ¿Cómo podemos hacer progresar las políticas? En C. Giné (coord), La educación inclusiva. (pp. 161-170). Barcelona: Horsori.

Alba, C. (2016). Educación inclusiva y enseñanza para todos: El diseno Universal para el aprendizaje. Madrid: Morata.

Arnaiz, P., de Haro, R., Maldonado, R.M. (2019). Barriers to Student Learning and Participation in an Inclusive School as Perceived by Future Education Professionals. Journal of New Approaches in Educational Research, 8(1),18-24.

Barrio, J.L. (2009). Hacia una Educación Inclusiva para todos. Revista Complutense de Educación, 20(1), 13-31.

Bauman, Z. (2007). Los retos de la educación en la modernidad líquida. Barcelona: Gedisa. 
Brynjolfsson, E. y McAfee, A. (2014). The Second Machine Age. Work, Progress, and Prosperity in a Time of Brilliant Technologies. Nueva York: Norton \& Company.

Cabero, J. (2014). Nuevas miradas sobre las TIC aplicadas en la educación. Andalucía Educativa, 81, http://www. juntadeandalucia.es/educacion/webportal/web/revista-andalucia-educativa/en-portada/-/noticia/detalle/nuevas-miradas-sobre-las-tic-aplicadas-en-la-educacion-julio-cabero-almenara-1 (3/03/2014).

Cabero, J. y Córdoba, M. (2009). Inclusión educativa: inclusión digital. Revista de Educación Inclusiva, 2(1), 61-77.

Cabero, J. y Valencia, R. (2018). Teacher education in ict: contributions from different training models. Revista Caribeña de Investigación Educativa, 2 (2), 61-76.

Cabero, J., y Ruiz-Palmero, J. (2018). Las Tecnologías de la información y la comunicación para la inclusión: reformulando la brecha digital. International Journal of Educational Research and Innovation, 9, 16-30

Cárdenas, M.C. y Anaya, C. (2018). @prende.mx: la política pública de educación digital de México para enfrentar los retos del siglo XXI. En En P. Rivera-Vargas, J. y otros (Eds.). Políticas Públicas para la Equidad Social (pp. 31-44). Santiago de Chile: USACH.

Casanova, M.A. (2017). Educación inclusiva en las aulas. Madrid: La Muralla.

Castro, R. y Blanco, A. (2009). Inclusión educativa y tecnologías de apoyo en discapacidad intelectual. Bordón. Revista de Pedagogía, 61(4), 93-108.

CEPAL (2015). Agenda digital para América Latina y el Caribe (eLAC2018). Santiago de Chile: CEPAL.

CEPAL (2018). Agenda 2030 y los Objetivos de Desarrollo Sostenible Una oportunidad para América Latina y el Caribe. Santiago de Chile: CEPAL.

Cullen, J.M., y Alber-Morgan, S.R. (2015). Technology Mediated SelfPrompting of Daily Living Skills for Adolescents and Adults with Disabilities: A Review of the Literature. Education and Training in Autism and Developmental Disabilities, 50(1).

Cummings S, Regeer B, de Haan L, Zweekhorst M, \& Bunders J. (2018). Critical discourse analysis of perspectives on knowledge and the knowledge society within the Sustainable Development Goals. Development Goals. Development Policy Review, 36, 727-742.

Escribano, A., y Martínez, A. (2013). Inclusión educativa y profesorado inclusivo. Madrid: Narcea.

Fernández-Batanero, J.M., Román, P. y El Homrani, M. (2017). TIC y discapacidad. Conocimiento del profesorado de educación primaria en Andalucía. Aula Abierta, 46, 65-72.

Fernández-Batanero, J.Mํa ${ }^{\mathrm{a}}$ Reyes, M.M. y El Homran, M. (2018). TIC y discapacidad. Principales barreras para la formación del profesorado. EDMETIC, Revista de Educación Mediática y TIC, 7(1), 1-25.

Floridi, L. (2017). The fourth revolution: how the Infosphere Is Reshaping Human Reality. Oxford: Oxford University Press.

Fundación Telefónica (2018). Estudio sobre la inclusión de las TIC en los centros educativos de aulas Fundación Telefónica. Madrid: Fundación Telefónica-OEI.

González, F. (2017). De la era de la perplejidad a la era de las oportunidades: finanzas para el crecimiento. En BBVA. La era de la perplejidad. Repensar el mundo que conocíamos (8-39). Madrid: Taurus.

Harari, Y. (2018). 21 lecciones para el siglo XXI. Barcelona: Debate. Lipovetsky, G. (2016). De la ligereza. Barcelona: Anagrama.

Lledó, E. (2018). Sobre la educación. Madrid: Taurus.
López, E., Vázquez, E. y Sarasola, J.L. (2015). Estudio bibliométrico de Pixel-Bit, Revista de medios y educación (2000-2013). Pixel-Bit. Revista de Medios y Educación, 46, 65-85.

Marchesi, A., Blanco, R., y Hernández, L. (coord.) (2014). Avances y desafíos de la educación inclusiva en Iberoamérica. Madrid: OEI.

Ministerio de Educación de la Nación (2017). Orientaciones pedagógicas de Educación Digital. Buenos Aires: Ministerio de Educación de la Presidencia de la Nación Argentina.

Moliner, O. (2013). Educación inclusiva. Castellón: Universitat Jaume I.

Morozov, E. (2013). La locura del solucionismo tecnológico. Madrid: Katz.

Moya, J. (2015). La educación como derecho. Madrid: La Muralla.

OECD (2015). Students, Computers and Learning. Making the Connection. París: OECD.

Orozco, G.H., Tejedor, F. y Calvo, M. (2014). Meta-análisis sobre el efecto del software educativo en alumnos con necesidades educativas especiales. Revista de Investigación Educativa, 35(1), 35-52.

Ortíz, A., Almanzan, L., Peñaherrera, M. y Cachón, J. (2014). Formación en tic de futuros maestros desde el análisis de la práctica en la Universidad de Jaén. Píxel-Bit. Revista de Medios y Educación, 44, 127-142.

Peñafiel, F. (2014). De la educación especial a la educación inclusiva, En Peñafiel, F., Torres, J.A. y Fernández, J.M. Evaluación e intervención didáctica, (pp. 13-38). Madrid: Pirámide .

Rangel, A. (2015). Competencias docentes digitales propuesta de un perfil. Pixel-Bit, Revista de Medios y Educación, 46, 235-248.

Rivera, P. y Cobo, C. (2018). Plan Ceibal en Uruguay: una política pública que conecta inclusión e innovación. En P. Rivera-Vargas, J. y otros (Eds.). Políticas Públicas para la Equidad Social. Santiago de Chile: USACH, 9-26.

Severin, E. (2016). Tecnologías digitales al servicio de la calidad educativa. Santiago de Chile: Unesco.

Starcic, A. y Bagon, S. (2014). ICT-supported learning for inclusion of people with special needs: Review of seven educational technology journals, 1970-2011. British Journal of Educational Technology, 45(2), 202-230.

Suárez, B. y López, A. (2018). Investigación en Educación Inclusiva: la producción de trabajos en Revistas Españolas y Tesis Doctorales. Revista Nacional e Internacional de Educación Inclusiva, 11(1), 151-174.

Toledo, P., Sánchez, y Gutiérrez, J.J. (2013). Evolución de la accesibilidad web en las Universidades Andaluzas. Pixel-Bit. Revista de Medios y Educación, 43, 65-83.

Ullman, H. (2017). Disability and public policy: institutional progress and challenges in Latin America. En Martínez, R. (ed). Institutional frameworks for social policy in Latin America and the Caribbean. Santiago de Chile, CEPAL, 255-278.

Unesco (2017). Sociedad digital: brechas y retos para la inclusión digital en América Latina y el Caribe. Montevideo: Unesco.

Valencia, R. (2018). Empleo del futuro. U-gob. Tecnología en Gobierno. https://u-gob.com/empleos-del-futuro/ (9/5/2018).

Valero, M. A. (2010). Tecnologías para la educación inclusiva: de la integración a la interacción. En P. Arnaiz, y otros (coord.). 25 Años de Integración Escolar en España: Tecnología e Inclusión en el ámbito educativo, laboral y comunitario. http://www.carm. es/web/pagina?IDCONTENIDO=6515\&IDTIPO=246\&RAS$\mathrm{TRO}=\mathrm{c} 943 \$ \mathrm{~m} 4331$.

Van Deursen, A. y Van Dijk, J. (2014). The digital divide shifts to differences in usage. New media \& Society, 16(6), 507-526.

West, D. (2016). Megachange. Economic Disruption, Political Upheaval, and Social Strife in the 21st Century. Washington: Brookings Institution. 
Zheng, B., Warschauer, M., Lin, C. y Chang, C. (2016). Learning in one-to-one laptop environments: A meta-analysis and research synthesis. Review of Educational Research, 86(4), 10521084. 